\title{
Changes in Polarization Position Angle across the Eclipse in the Double Pulsar System
}

\author{
R. Yuen ${ }^{1,2}$, R. N. Manchester ${ }^{1}$, M. Burgay ${ }^{3}$, F. Camilo ${ }^{4}$, M. Kramer ${ }^{5}$, \\ D. B. Melrose ${ }^{2}$ and I. H. Stairs ${ }^{6}$ \\ ${ }^{1}$ CSIRO Astronomy and Space Science, Australia Telescope National Facility, P.O. Box 76, \\ Epping, NSW 1710, Australia \\ ${ }^{2}$ SIfA, School of Physics, the University of Sydney, NSW 2006, Australia \\ ${ }^{3}$ INAF-Osservatorio Astronomica di Cagliari, Loc. Poggio dei Pini, Strada 54, 09012 \\ Capoterra, Italy \\ ${ }^{4}$ Columbia Astrophysics Laboratory, Columbia University, New York, NY 10027, USA \\ ${ }^{5}$ Max Planck Institut für Radioastronomie, Auf dem Hügel 69, 53121 Bonn, Germany \\ ${ }^{6}$ Department of Physics and Astronomy, University of British Columbia, Vancouver, BC V6T \\ 1Z1, Canada
}

\begin{abstract}
We investigate the changes in polarization position angle in radiation from pulsar A around the eclipse in the Double Pulsar system PSR J0737-3039A/B at the $20 \mathrm{~cm}$ and $50 \mathrm{~cm}$ wavelengths using the Parkes $64-\mathrm{m}$ radio telescope. The changes are $\sim 2 \sigma$ during and shortly after the eclipse at $20 \mathrm{~cm}$ but less significant at $50 \mathrm{~cm}$. We show that the changes in position angle during the eclipse can be modelled by differential synchrotron absorption in the eclipse regions. Position angle changes after the eclipse are interpreted as Faraday rotation in the magnetotail of pulsar B. Implied charge densities are consistent with the Goldreich-Julian density, suggesting that the particle energies in the magnetotail are mildly relativistic.
\end{abstract}

Keywords. binaries: eclipsing — polarization — pulsars: individual (PSR J0737-3039A, PSR J0737-3039B)

\section{Introduction}

The almost edge-on orbit in the Double Pulsar system, which consists of two pulsars (pulsar A and pulsar B), results in a 30-second eclipse observable once each orbit when pulsar A moves behind pulsar B at orbital longitude of $\sim 90^{\circ}$ measured from the ascending node (Lyne et al. 2004). The magnetosphere of pulsar B is truncated because of the strong relativistic wind from pulsar $\mathrm{A}\left(\dot{E}_{A} / \dot{E}_{B} \sim 3500\right)$ forming a magnetotail that probably reaches a distance of several semi-major axes of pulsar B's orbit (the semi-major axis is $4.5 \times 10^{8} \mathrm{~m}$ ) (Arons et al. 2004). Because of the orbital motion, the magnetotail is curved behind pulsar B. Across the eclipse regions, pulsar A's radiation passes through pulsar B's truncated magnetosphere and interacts with the plasma during an eclipse, then, as the two pulsars advance in orbit away from the superior conjunction, pulsar A's radiation begins to traverse the magnetotail. In this paper, we investigate the changes in polarization position angle in radiation from pulsar A during and around the eclipses.

\section{Data analysis and Results}

The data of interest were taken at $20 \mathrm{~cm}$ and $50 \mathrm{~cm}$ using the Parkes $64-\mathrm{m}$ radio telescope with sub-integration time of $30 \mathrm{~s}$. The PSRCHIVE software suite (Hotan et al. 2004) was used for data analysis including interference rejection and calibration. Data were 


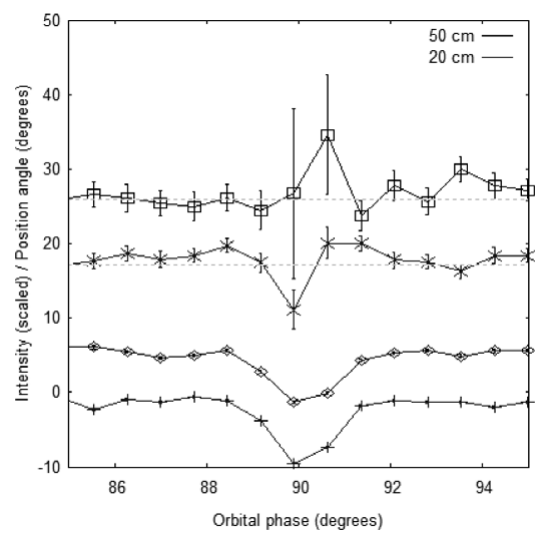

Figure 1. Plot of polarization position angle in degrees (upper curves) and Stokes I (total intensity) parameter (lower curves) plotted against orbital phase in degrees for $50 \mathrm{~cm}$ (black curve) and $20 \mathrm{~cm}$ (blue curve) at $\sim 18 \mathrm{~s}$ time resolution. The curve for position angle at $50 \mathrm{~cm}$ is offset by $3^{\circ}$ for clarity. The Stokes I curves clearly show the eclipse centred near $90^{\circ}$. The horizontal lines represent average baseline values.

summed in frequency using the corrected rotation measure, $+112.3 \mathrm{rad} \mathrm{m}^{-2}$ (Manchester et al. 2010). We obtained 38 files at $20 \mathrm{~cm}$ and 13 at $50 \mathrm{~cm}$ with good quality data. To improve the signal-to-noise ratio, we summed the files in each band, binning the results in orbital phase with phase bins of $\sim 0.7^{\circ}$ or $\sim 18 \mathrm{~s}$ in width. Finally, Stokes parameters were summed across the main pulse and interpulse to form the average Stokes parameters for each orbital phase bin.

We consistently detected changes in position angle during and shortly after an eclipse at both wavelengths (Figure 1). Inspection of the $20 \mathrm{~cm}$ curve in the eclipse region shows that the position angle decreases during the ingress phase then rises to its maximum value during egress phase with a change of approximately $\sim 6^{\circ} \pm 3^{\circ}$ and $\sim 3^{\circ} \pm 2^{\circ}$ respectively relative to the mean baseline level. It remains high for the following phase bin, then drops back to the baseline value. There are also changes in polarization angle in the corresponding regions during the eclipse at $50 \mathrm{~cm}$, but the uncertainties are larger. Nevertheless, the position angle changes shortly after the eclipse are consistent with those at $20 \mathrm{~cm}$. We confirmed our results by randomly partitioning the $20 \mathrm{~cm}$ files into two, three or four subsets either randomly or chronologically, which show similar patterns, i.e., a decrease in position angle during ingress followed by an increase during egress.

\section{Mechanisms to change the polarization position angle}

Eclipse region: differential synchrotron absorption. With our 18s averaging time, the direction of the differential synchrotron absorption relative to the incoming radiation can be approximated by averaging the magnetic field perpendicular to the rotation and magnetic axes to zero leaving a net magnetic field along B's rotation axis. The position angle of the transmitted radiation changes with increasing optical depth, $\tau_{\nu}=\alpha_{\nu} L$, with the position angle moving toward the angle of the projected magnetic field. Here $L$ is the effective path length through the absorbing medium and $\alpha_{\nu}$ is the polarization-averaged synchrotron absorption coefficient for a relativistic thermal distribution of particles (Lyutikov \& Thompson 2005). Denoting $\chi_{0}$ and $\chi_{\nu}$ to be the incident and emergent polarization angles relative to the projected magnetic field direction, and assuming $\chi_{0}=45^{\circ}$ gives an order of magnitude change in polarization or position angle, 
$\Delta \psi=\chi_{\nu}-\chi_{0}$, of $10^{\circ}$ at $20 \mathrm{~cm}$. This is consistent with our measurements suggesting that differential synchrotron absorption in the closed field-line regions can plausibly account for the observed position angle changes during the eclipse.

Magnetotail: Faraday rotation. Net magnetic field in the line of sight results from the large inclination angle in pulsar B and the misalignment between its rotation axis and the orbital normal, which result in an asymmetric field structure in the magnetotail as the pulsar rotates within the confining magnetosheath (Spitkovsky and Arons 2004). Assuming magnetic field scales as $r^{-2}$ up to $L / 2$ and as $r^{-1}$ at larger distance in the magnetotail ( $L \approx 1.5 \times 10^{-8}$ pc is the semi-major axis of pulsar B's orbit), and with $\Delta \psi_{50}=0.81 L\left\langle n_{e} B\right\rangle \lambda^{2} \sim-5^{\circ}$, gives $n_{e} \sim 40 \mathrm{~cm}^{-3}$, which is comparable to the estimated corotation charge density (Goldreich and Julian 1969) of $n_{e} \sim 20 \mathrm{~cm}^{-3}$. This suggests that Faraday rotation may be responsible for the changes in position angle away from the eclipse region, which also implies that the charged particles are no more than mildly relativistic. Direct generation of mildly relativistic pairs is favored for magnetic fields with $0.02 B_{\text {cr }}<B<0.1 B_{\mathrm{cr}}$, where $B_{\mathrm{cr}} \approx 4.9 \times 10^{13} \mathrm{G}$ is the critical field strength (Weise \& Melrose 2002).

\section{Conclusions}

We have observed changes in polarization position angle in radiation from pulsar A during and shortly after the eclipse in the Double Pulsar system at $20 \mathrm{~cm}$ and $50 \mathrm{~cm}$ wavelengths. These changes can be accounted for by differential synchrotron absorption and Faraday rotation respectively with the latter implying that pairs must be created directly in nonrelativistic regime.

\section{Acknowledgments}

We thank our colleagues for assistance with the observations reported in this paper. The Parkes radio telescope is part of the Australia Telescope National Facility which is funded by the Commonwealth of Australia for operation as a National Facility managed by CSIRO. Pulsar research at UBC is supported by an NSERC Discovery Grant.

\section{References}

Arons, J., Backer, D. C., Spitkovsky, A., \& Kaspi, V. M. 2004, Proc. 2004 Apsen Winter Conf. on Astrophysics, Binary Radio Pulsars, San Francisco: ASP

Goldreich, P. \& Julian, W. H. 1969, ApJ, 157, 869

Hotan, A. W., van Straten, W., \& Manchester, R. N. 2004, Publications of the Astronomcial Society of Australia, 21, 302

Lyne, A. G., Burgay, M., Kramer, M., Possenti, A., Manchester, R. N. et al. 2004, Science, 303, 1153

Lyutikov, M. \& Thompson, C. 2005, ApJ, 634, 1223

Manchester, R. N., Kramer, M., Stairs, I. H., Burgay, M., Camilo, F., Hobbs, G. B., Lorimer, D. R., Lyne, A. G., McLaughlin, M. A., McPhee, C. A., Possenti, A., Reynolds, J. E., \& van Straten, W. 2010, ApJ, 710, 1694

Spitkovsky, A. \& Arons, J. 2004, Bulletin of the American Astronomical Society, HEAD meeting, $36(8,08.02), 917$

Weise, J. I. \& Melrose, D. B. 2002, MNRAS, 329, 115 